\title{
Application of the Concept of Presentational Understanding in the Practice Courseware of Electric Power Maintenance
}

\author{
Wenquan Ma*, Hongwei Qin, Yi You, Huaxiao Ding \\ Dalian Training Center of State Grid Liaoning Electric Power Co., Ltd
}

\begin{abstract}
Currently, the level of intelligent construction in the power industry is constantly improving, and power maintenance has gradually realized intelligent control and management, which greatly guarantees the operational safety of smart grids. In order to solve the problem that traditional courseware cannot meet the practical requirements of electric power maintenance, a kind of practical electric power maintenance courseware is researched and designed based on the concept of presentational understanding. From the perspective of improving the real-time and accuracy of power overhaul, the monitoring information of the substation is effectively presented. On this basis, effective inspection and repair work are carried out on the secondary circuit of the substation. The content of the courseware enhances the deeper understanding of the electric power maintenance staff.
\end{abstract}

\section{Introduction}

When there is a failure during the operation of power facilities, the secondary circuit will automatically alarm to maintain the stable operation of the substation [1]. However, due to the interference of the on-site environment, the secondary loop alarm function is difficult to effectively distinguish between relay protection abnormalities and other abnormal conditions, resulting in the failure of substation operation failures to be alerted and eliminated in time. Therefore, it is necessary to adopt more scientific and effective methods for the training of power maintenance work to help maintenance personnel improve their technical level [2].

Therefore, in order to meet the training needs of the current power maintenance work, reduce the gap between the training environment and the actual operating environment, and improve the training effect, a training courseware in the paper is designed based on the concept of presentational understanding, through a multi-level structure of the courseware design and model the virtual scene of the substation to achieve the effect of visualized power facility maintenance scenes and provide effective guarantee for improving training efficiency.

\section{Substation Monitoring Information Presentation}

Since there is more information about the secondary circuit monitoring in the substation, in order to ensure the accuracy of the online monitoring, the monitoring information of the substation needs to be presented [3]. VR technology is used to simulate the actual situation of the substation, collect the environmental information of the entire substation, and the acquired data is used to establish the corresponding virtual environment model, complete the drawing of the graphic styles of each element such as the circuit connection of each device and the receiving plate, and then change the information of each substation. The secondary circuit data connection generates the secondary circuit display screen, and the preliminary planning of the substation secondary circuit monitoring process is carried out according to the simulation results [4].

Due to the large amount of state to be monitored, different characteristics of the detection state parameters need to be judged, so the monitoring information of the substation is presented [5]. The attributes and services of information presentation are shown in figure 1.

1850508590@qq.com 


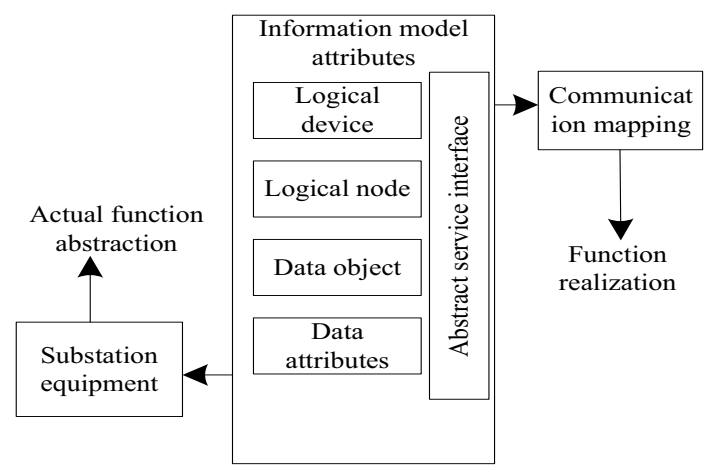

Figure 1 The attributes and service content of information presentation

According to the idea of hierarchical modeling, the attributes in the monitoring information are divided into four aspects, namely logical devices, logical nodes, data objects and data attributes. The hierarchical structure is shown in figure 2 .

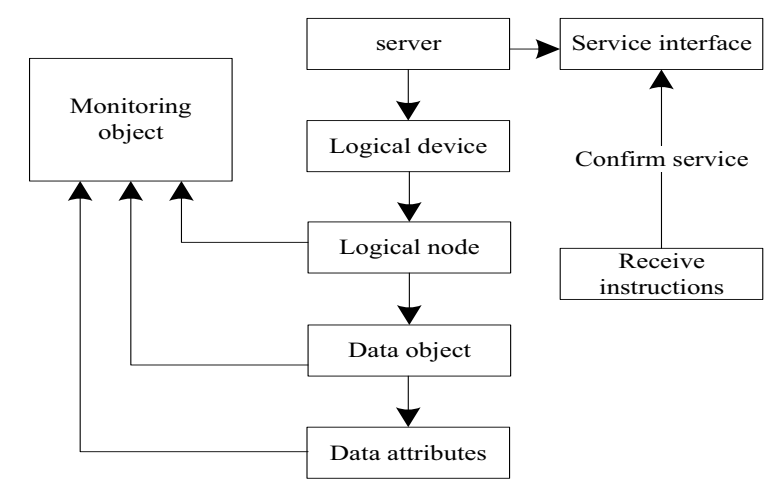

Figure 2 Schematic diagram of information attribute hierarchy

\section{Scene Modeling}

Taking the actual layout and characteristics of the substation as a reference, a progressive presentation method is used to simulate the scene of the courseware to create a virtual substation scene. First, the scene information is presented, and its membership degree is analyzed based on the principle of discriminant analysis, and the substation scene information association domain is established [6]. On this basis, the scene state judgment index value is established, and the process is as follows:

$$
Z=\delta \times \frac{c}{V}
$$

Where $V$ represents the setting value of the scene state of the substation, the value range is between 0 and 1 , $c$ represents the measured value of the scene safety index, and $\delta$ represents the ratio of the measured value of the scene safety index of the operation process to the rated state standard [7].

The virtual scene of the substation includes roaming scene, space scene and operation scene. Among them, the roaming scene can display the appearance information of primary electrical equipment, protection screens and control rooms on site; the spatial scene refers to the overall environment of the substation, such as the environmental information of the substation under different natural environmental factors such as sunny and cloudy and rainy days; the operating scene is Refers to the operation process scene of each electrical equipment, such as the process scene of checking and repairing the main transformer [8].

Figure 3 shows the overall overhaul scene of the substation. The overhaul process of the substation can be completed with the assistance of a joystick, mouse and keyboard [9]. The action characteristics of electrical equipment such as circuit breakers can be seen through the maintenance process, which greatly enhances the realism of the substation scene.

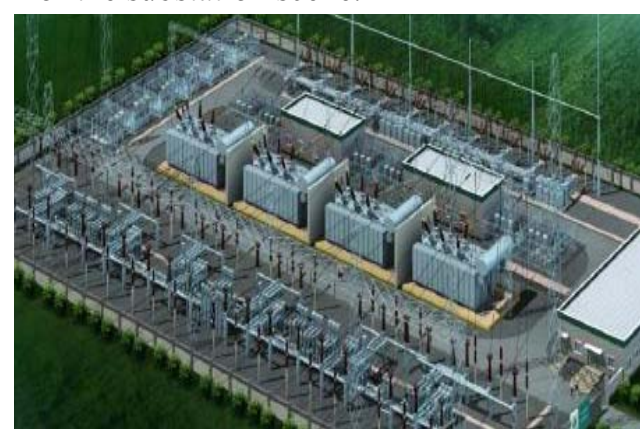

Figure 3 Overall overhaul scenario of substation

Since substation equipment is extremely susceptible to the influence of the external environment during operation, it is necessary to determine whether there is equipment failure information in the status scene information. Assuming that the rated warning value is set to 1 , the process of calculating the security level of the 
power equipment status in the virtual scene of the substation is as follows:

$$
Y=Z \times \frac{\gamma \mu}{V}
$$

Where $\gamma$ represents the fault identification parameter. When there is a clear fault, the value $\gamma$ is 0 , and when there is no clear fault, the value $\gamma$ is $1 ; \mu$ represents the rated power of the power equipment. When $Y>1$, it means that there is a serious fault problem in the scene. When $Y=1$, it means that there is a hidden trouble in the scene, and it needs to be checked and corrected by professionals in time. VR technology can be used to identify the identifier of the power equipment during the inspection, and the fault judgment and diagnosis can be realized through the intelligent information courseware of the substation. When $Y<1$, it means that the scene status is safe [10].

\section{Training Module Design}

Power failures will inevitably occur in substations during operation. Therefore, in order for operators to quickly solve substation equipment, wiring and other faults and maintain the normal operation of virtual substations, different subject modules of training courses are designed, and the interaction relationship between the subjects is shown in figure 4.

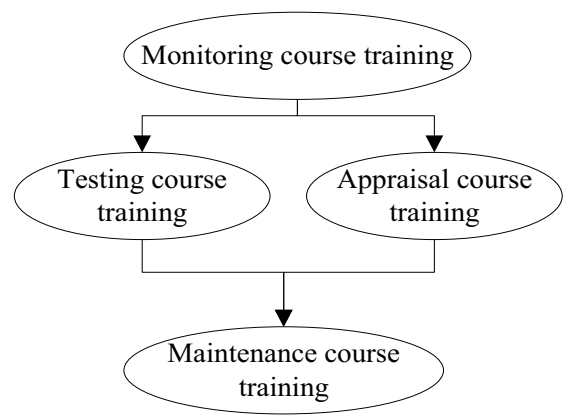

Figure 4 Interaction process diagram of training topics

The interaction process of training topics is that:

(1) Monitoring course training: If there is a fault in the substation during operation, the training content is responsible for outputting fault current and voltage signals; in other cases, the training content is responsible for comprehensive monitoring and analysis of substation equipment, operating mechanism, DC courseware and Relay protection and other devices.

(2) Testing course training: Effectively deal with the testing of power equipment. If there is a faulty equipment, it needs to be repaired.

(3) Appraisal course training: Appraise whether there are status changes in power lines and equipment according to the processed signals, and feed them back to the equipment maintenance work if there are changes.

(4) Maintenance course training: study the faults of power facilities, and repair the specific faults.

\section{Experimental Comparison and Analysis}

\subsection{Experimental Method}

The specific electric power maintenance practice course will take the substation as an example, and the steps for the fusion of monitoring information and the way of understanding are as follows:

(1) The function of substation equipment is abstracted and decomposed, and the secondary circuit information of the substation is regarded as a logical node. According to the dsfo-7-4 standard, a number of relatively independent logical nodes that can complete its basic functions are abstracted, and data related to each logical node is abstracted. The object is defined and the calculation process is as follows:

$$
G=\frac{T_{0}}{A \times S}
$$

In formula 3, $G$ represents the measured value of the substation device, $A$ represents the information data collection of the detected substation, $S$ represents the complex monitoring object, and $T_{0}$ represents the online monitoring time of the secondary circuit of the substation at a certain moment.

Through the above decomposition results, logical nodes and corresponding data objects are obtained.

(2) Abstract and identify the entire equipment information, and decompose and classify the actual functions of the equipment. Since the logical equipment in the substation contains logical nodes with different functions and services corresponding to the logical nodes, the calculation process for the data processing of logical nodes with different functions is as follows:

$$
H=\sum_{x} i \times \frac{q}{f_{e}}
$$

In formula $4, H$ represents the monitoring data of different monitoring objects, $\sum_{x} i$ indicates the monitoring information of the monitoring objects $i$, $f_{e}$ refers to the type of monitoring information, and $q$ is the monitoring classification factor.

(3)Determine the communication service, organize the attributes and services obtained above in the form of classes to form a deterministic information exchange model.

When using the information exchange model, define the functions to be implemented by each module and define the required data types. According to the definition results, complete the presentive fusion of maintenance based on substation monitoring information.

\subsection{Analysis of Experimental Results}

Table 1 shows the statistical results of the response time of different teaching methods to the results of electric 
maintenance courses.

Table 1 Statistical results of reaction time of different teaching methods in electric maintenance courses $\left(\times 10^{3} \mathrm{~s}\right)$

\begin{tabular}{ccccc}
\hline $\begin{array}{c}\text { Number of } \\
\text { iterations/time }\end{array}$ & $\begin{array}{c}\text { Method } \\
\text { Document }\end{array}$ & $\begin{array}{c}\text { Literature [3] } \\
\text { method }\end{array}$ & $\begin{array}{c}\text { Literature [4] } \\
\text { method }\end{array}$ & $\begin{array}{c}\text { Literature [5] } \\
\text { method }\end{array}$ \\
\hline 10 & 6.34 & 8.32 & 9.79 & 9.82 \\
20 & 7.98 & 8.47 & 9.03 & 9.46 \\
30 & 7.63 & 7.94 & 10.54 & 9.94 \\
40 & 6.19 & 9.01 & 10.75 & 10.59 \\
50 & 7.96 & 9.27 & 9.88 & 9.73 \\
\hline
\end{tabular}

In Table 1 , as the number of iterations of the test process changes, the maintenance response time of different methods also changes constantly. However, the response time of using the proposed method for substation monitoring information to repair is always kept to the minimum, and the maximum response time is only $7.98 \times 103 \mathrm{~s}$, which is far less than the other three monitoring methods. Therefore, it can be proved that the proposed maintenance practice course based on the presentational understanding concept has strong real-time performance for substations, and can quickly make judgments on substation faults.

In the test process, the accuracy of the maintenance effect can be evaluated by monitoring the accuracy of the maintenance process. The accuracy of the maintenance process is directly proportional to the accuracy of the maintenance effect. The higher the accuracy of the maintenance process, the higher the accuracy of the maintenance effect. Figure 5 shows the comparison result of the action accuracy of the maintenance process of different teaching methods.

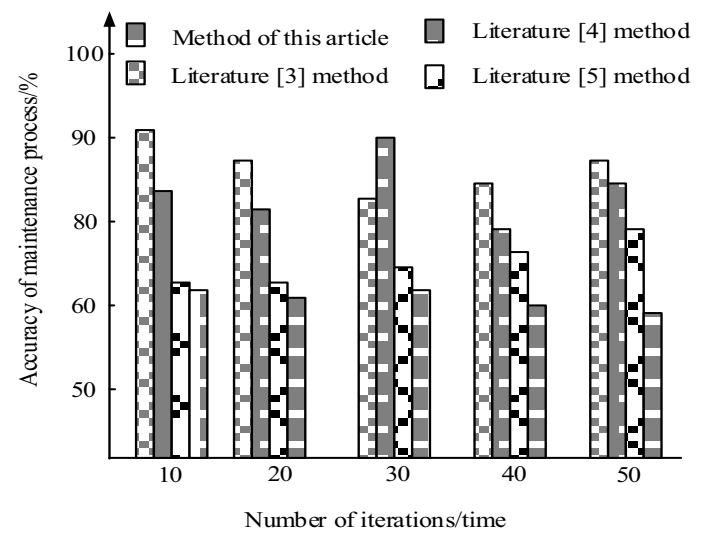

Figure 5. Comparison results of action accuracy in maintenance process of different teaching methods

In Figure 5, as the number of test iterations increases, the accuracy of the maintenance process actions of different methods also changes. When the number of iterations is small, the accuracy of the maintenance process of the method in literature [5] is similar to that of the method in this paper, but when the number of iterations reaches 30 times, the accuracy of the maintenance process of the method in literature [5] shows an upward trend, gradually higher than that of The proposed method. However, the accuracy of the maintenance process action of the methods in literature [3] and literature [4] is always significantly higher than the proposed method. Therefore, the test results can show that the proposed power maintenance practice courseware based on the presentational understanding concept has high practical application value.

\section{Conclusion}

The presentational understanding concept can be used in the power maintenance practice courseware to realize the maintenance of power facilities through progressive presentation processing, and can perform operations or animation processing for a single electrical appliance. Corresponding to each characteristic information point of the model, trainees experience the substation maintenance scene more realistically during training, and can more fully control the substation structure. Through the specific setting of the maintenance content, the monitoring and maintenance of the substation equipment can be effectively realized.

\section{References}

1. Qian Ping, Zhang Yong, Xu Jieming. Research on terminal visual inspection technology of smart grid inspection robot $[\mathrm{J}]$. Modern Electronic Technology, 2018(18): 113-116.

2. Wu Yichun, Zhou Lei, Fang Xuelei, et al. Development and application of virtual simulation training system for substation switchgear maintenance[J]. Electrical Engineering Technology, 
2019,467(5): 54-56.

3. Li Cheng, Lu Yujun, Wang Ning, et al. Design and implementation of remote virtual terminal access system for smart substation[J]. Electrical Measurement \& Instrumentation, 2016, 53(13): 123-128.

4. Jiang Long, Zhang Degang, Li Zehe, etc. Design and implementation of immersive substation simulation training system based on virtual reality helmet[J]. Electrical Engineering Technology 2017, 4(11): 46-48.

5. Sun Ninghui, Tan Guangming. Development and policy of high performance computer [J]. Bulletin of Chinese Academy of Sciences, 2019,34:609-616.

6. Shen Xiaoteng. The ecological transformation and innovation strategy of ideological and political education in the era of artificial intelligence [J]. Future and Development, 2019, 43: 97-102.

7. Zhao Jianbing. Analysis of the effective application of multimedia resources on electronic whiteboards[J]. Contemporary Educational Practice and Teaching Research, 2015,9: 12-18.

8. Zhao Dongying. Research on traditional teaching methods at the basic education stage[J]. Tomorrow, 2018,21:39-45.

9. Fu Yujuan, He Junshi, Zhang Xudong, Zhang Jing. Discussion on Modern Teaching Methods [J]. China Science and Technology Information, 2012, 11:230-240.

10. Jang S J. Integrating the interactive whiteboard and peer coaching to develop the TPACK of secondary science teachers[J]. Computers \& Education, 2010, $55: 17-23$ 